\begin{abstract}
Purpose - Although there is a growing evidence base for the value of psychosocial and artsbased strategies for enhancing wellbeing amongst dementia patients, relatively little attention has been paid to literature-based interventions. This service evaluation assesses the impact of Shared Reading (SR) groups, a programme developed and implemented by The Reader Organisation, on quality of life for care home residents living with mild/moderate dementia. Design/methodology/approach - Thirty six individuals were recruited from four care homes, which were randomly assigned to either reading-waiting groups (three months reading, followed by three months no reading) or waiting-reading groups (three months no reading, followed by three months reading). Quality of life was assessed by the DEMQOLProxy.
\end{abstract}

Findings - Compared to the waiting condition, the positive effects of SR on quality of life were demonstrated at the commencement of the reading groups and were maintained once the activity ended. Low levels of baseline psychopathological symptoms prevented analyses on whether the intervention impacted on the clinical signs of dementia.

Limitations - Limitations included the small sample and lack of control for confounding variables.

Originality/value - The therapeutic potential of reading groups is discussed as a positive and practical intervention for older adults living with dementia.

Keywords: Dementia, Quality of Life, Reading Therapy Article Type: Research paper 


\section{An Evaluation of Shared Reading Groups for Adults Living with Dementia: Preliminary Findings}

Dementia not only exerts a devastating impact on individuals and families, but is associated with a substantial financial burden that is projected to reach $£ 27 \mathrm{bn}$ in the UK by 2018 (Department of Health [DoH], 2012a). In turn, global prevalence is steadily expanding in middle- and high-income countries, and is expected to nearly double every 20 years to 115 million cases by 2050 (Alzheimer's Disease International, 2012). The importance of prioritising evidence based treatments, including the need to promote quality of life for patients and their carers, is increasingly recognised by policy makers ( $\mathrm{DoH}, 2012 \mathrm{~b})$, with a growing consensus that identifies 'living well' as an important outcome for evaluating interventions. Consonant with more general recommendations around arts and health strategies (Staricoff, 2004), consideration has therefore been paid to a range of participatory, non-pharmacological interventions for dementia patients, including music therapy (Svansdottir \& Snaedal, 2006), comedy (Low et al., 2013), and art programmes (Kinney \& Rentz, 2005). For example, the storytelling intervention TimeSlips, which emphasises improvisation and creative expression as opposed to reminiscence, has been associated with a range of positive gains amongst adults living with dementia, including increased pleasure scores on the Observed Emotion Rating Scale, small to moderate improvements in social and treatment needs communication (Philips et al., 2013), enhanced quality of life outcomes (George \& Houser, 2014), increased alertness and engagement (Fritsch et al., 2009), and improvements in neuropsychiatric symptoms (Gerdner, 2000). Like reading groups, an important aspect of TimeSlips is its emphasis on spontaneous narrative and self-expression (independent of cognitive ability) within a supportive communal setting. However, despite recommendations that even patients with advanced dementia can engage with reading if material is presented in an accessible format (Alzheimer's Disease International, 2011), there 
has been relatively little exploration of literature-based strategies within dementia care. As such, this pilot evaluation addresses the impact of a specific intervention, Shared Reading (SR), for enhancing health and well-being outcomes amongst individuals living with dementia.

\section{The Intervention}

SR is devised and implemented by The Reader Organisation (TRO), an award-winning charitable social enterprise that develops spaces in which people can relate both with serious literature and with one another. Groups are run by TRO-trained project workers, who facilitate dynamic open-ended discussions of a text and participants' personal responses to it. Specifically, SR differs from the format of traditional reading groups in that material is unseen in advance and communally read aloud during the session by a project worker. The intervention has been successfully implemented in a range of settings and populations, including: prisons (Billington, 2011); socially disadvantaged adults (e.g., those who are vulnerably housed or recovering from substance dependence: Hodge et al., 2007); and patients experiencing depression (Dowrick et al., 2012), chronic pain (Billington et al., 2014), and neurological conditions (Robinson, 2008). In reviewing available evidence for SR, Dowrick et al. (2012) argue that the approach "harnesses the power of reading as a cognitive process, but also acts as a powerful socially coalescing presence" (p.16), with cited positive outcomes covering the emotional, cognitive and interpersonal (Longden et al., 2015).

TRO currently administers 35 reading groups in the UK for elderly adults with and without dementia, which operate in such diverse venues as care homes, hospitals, sheltered housing, and day centres. Initial experimental work is intriguing, but meagre. Specifically, assessment of 61 service-users and 20 staff members found that six months of SR was associated with clinical improvements on the Neuropsychiatric Inventory Questionnaire 
(NPI-Q; Kaufer et al., 2000), with supporting qualitative interviews identifying three formative themes for effectiveness: 1) the components of the intervention itself (e.g., stimulating content, supportive staff presence); 2) enjoyment, authenticity, meaningfulness and renewed sense of personal identity; and 3) enhancement of listening, memory and attention (Billington et al., 2013). In turn, qualitative analysis of sessions with 40 care home residents indicated enjoyment, stimulation, and a sense of mastery and accomplishment (Centre for Research into Reading, Literature and Society: CRILS, 2014). Further benefits cited by staff members included social and cognitive gains, as well as the intervention's capacity to promote interaction between residents and workers, with carers likewise praising the opportunities for communication and relational contact. However, while these previous studies provide qualitative data around quality of life benefits, there is currently a lack of evidence for a quantifiable effect of SR in this domain.

Furthermore, while reading habits have been correlated with cognitive impairments in adults with age-related memory decline (Juncos-Rabadan et al., 2012), research in healthy adult populations has also shown that neural processing when reading complex poetry has the potential to galvanise brain pathways and to influence emotion networks and memory function (Davis, 2008; Davis et al., 2013; Thierry et al., 2008). In turn, a longitudinal cohort study of 294 individuals (mean follow-up 5.8 years) found that self-reported cognitive activity levels across the lifespan (e.g., reading, visiting libraries, letter writing) was associated with slower rates of cognitive decline in each year prior to death, independently of common neuropathologic conditions (Wilson et al., 2013).

Taken together, there are grounds for inferring a beneficial role for SR engagement in dementia care and a rationale for developing the evidence base for its application and methods of impact. As such, this pilot evaluation aimed to quantify the impact of SR on care hone residents living with dementia in terms of both behavioural symptoms and/or quality of 
life. It was hypothesised that reading group activity would be associated with improvements in both domains.

\section{Method}

\section{Participants}

The sample was recruited from four residential care homes in the Wirral area of Merseyside and comprised 36 individuals with mild to moderate dementia. Dementia classification was not assessed with formal rating scales, but identified to the researchers by care home managers on the basis of their clinical expertise. Over the course of the intervention one resident died and two withdrew. Two residents who joined the project after baseline were also not included, leaving 31 datasets in the final analysis for a small to medium effect size (.25) and statistical power of .77. Sixteen participants were female. However, data concerning other characteristics (e.g., age, time since diagnosis, duration of care home residence) was not collected, as it was not related to the primary concern of evaluating SR impact.

Due to the exploratory nature of the project, we did not have formal eligibility criteria. Thus while some residents were approached by care home staff because they were known to have enjoyed reading in the past (e.g., a former teacher), the ultimate choice to attend groups was made by residents themselves. However, several reasons for exclusion were identified in liaison with care home staff, namely: residents who had violent/disruptive tendencies; those who were likely to become distressed or agitated in a group setting; and those who were unable to sit still for a reasonable period of time.

\section{Measures}

Because the project was concerned with the perceived impact of SR from those who cared daily for the participants (and would thus be likely to notice any changes attributable to the group itself as opposed to other situational factors), quality of life was assessed with a third- 
party measure. The DEMQOL-Proxy (Smith et al., 2005) is an interviewer-administered carer-rated measure that demonstrates robust psychometric properties amongst individuals with mild/moderate dementia (Rowen et al., 2012; Smith et al., 2005). The instrument evaluates various aspects of mood (e.g., contentment, energy levels, cheerfulness), exhibited concern over memory difficulties, and participation levels in everyday activities (e.g., cleanliness, finances, sociability).

Behavioural symptoms were assessed with the brief clinical form of the Neuropsychiatric Inventory Questionnaire (NPI-Q; Kaufer et al., 2000), a clinical scale that measures the presence and severity of: delusions; hallucinations; agitation/aggression; anxiety; depression/dysphoria; elation/euphoria; apathy/indifference; disinhibition; irritability/lability; and motor disturbance. Two neuro-vegetative areas are also assessed: night-time behaviour and appetite. The NPI-Q is widely applied in evaluating dementiarelated psychopathology and has proven reliability and validity (Kaufer et al., 2000). In the current project, only symptom severity data were collected as the DEMQOL-Proxy incorporates the emotional aspects of living with dementia.

\section{Procedure}

Of the four participating care homes, two were randomly assigned to the reading-waiting group while the remaining two were assigned to the waiting-reading group. All data were collected from care home staff at baseline and then every month thereafter for six months. Qualitative data were also gathered via semi-structured interviews with residents, care staff, relatives and TRO project workers, the results of which are presented by CRILS (2014). Reading groups were facilitated by TRO project workers, with the following adaptations made to the SR model to make the experience more meaningful and accessible for people living with dementia: 
1. Session duration was shortened from 90 minutes to one hour and project workers read material in a louder tone than is customary as well as taking more time to describe text content before proceeding to discussion.

2. Poems were employed in preference to narrative prose, as the language tends to be more compressed and salient (thus comprising what neuropsychologists refer to as 'engrams'; a representation of a whole experience analogous to one's own, thereby encoding a memory trace). Rhyme and rhythm may further encourage and sustain concentration, and reproducing poems on a single page make it less likely that participants will lose their place. Participants were additionally of a generation often required memorise poems at school, and it is often the case that even individuals with severe dementia are able to flawlessly recite poems learnt in childhood.

3. Discussion questions were chosen with care to avoid discomfort from a lack of immediate comprehension, and queries requiring participants to draw on memories and past experiences were generally avoided. However, while the literature may often evoke past memories and encounters, SR is not a reminiscence project. As stated by CRILS (2014, p.4) "members are encouraged to enjoy the literature as an experience in the present moment as much as a medium for reflecting on past experiences, and the reading material selected therefore has to be able to stand alone as a moment in itself so that members are free to enjoy it however they choose.” Examples of selected poems included 'Daffodils' by William Wordsworth, 'In Flanders Fields' by John McCrae, and 'Love and Friendship' by Emily Brontë.

Group size varied from six to ten participants, with a care worker in attendance to provide support, safeguard welfare, and to obtain direct experience of the project. To ensure 
group members were treated according to the experience of the encounter, TRO project workers had no prior knowledge about residents' clinical status.

As this study was deemed to be a service evaluation by the local National Health Service (NHS) research ethics committee, formal National Research Ethics Service (NRES) approval was not required, although the project was approved by the local NHS Trust Research and Development office. The ethical decision was made on the basis that the project evaluated current care (SR groups commissioned by the local Primary Care Trust were already in operation); no member of the study team was involved with residents' direct care; the groups were run by an independent worker not involved in the study; no information was collected from case notes or medical records; and there was no process of randomization for individual participants.

- Table 1 here -

\section{Results}

Mean DEMQOL-Proxy scores according to reading or waiting condition are depicted in Table 1. Intervention impact was assessed using a repeated measures analysis of variance, comparing DEMQOL-Proxy scores in the two conditions (waiting then reading [ $n=14]$ versus reading then waiting $[\mathrm{n}=17])$. There was a significant group $\mathrm{x}$ time interaction $(\mathrm{F}(1,29)=$ $14.588, p<.001)$. Mean scores for participants in the 'waiting then reading' group rose from $102.9(\mathrm{SD}=11.7)$ to $114.9(\mathrm{SD}=3.0)$, whereas those in the 'reading then waiting' group remained steady; with a mean score of $114.3(\mathrm{SD}=2.4)$ in the 'reading' period and 115.8 $(\mathrm{SD}=2.7)$ in the subsequent 'waiting period'.

Owing to extremely low NPI-Q scores (i.e., the vast majority of participants reported no distressing behavioural symptoms for the duration of the evaluation) these data were not analysed further. 


\section{Discussion}

The current findings, combined with existing qualitative (Billington et al., 2013; CRILS, 2014) and quantitative (Billington et al., 2013) data can only be considered tentatively in the absence of larger-scale, more controlled studies. Nevertheless, a quantifiably positive effect on quality of life was demonstrated at SR's commencement in comparison to the waiting condition, and appears to have been maintained once the activity ended. These results indicate that literature-based interventions may have value for individuals living with dementia, and add to an existing evidence base for the role of arts-based interventions for augmenting wellbeing in this population.

This evaluation does not permit definite conclusions about SR's mechanisms of impact, although it is possible to speculate on the basis of existing literature. Social contact, for example, is known to be an important requirement for dementia patients (van der Roest et al., 2007), and SR provides a supportive forum in which residents can engage in meaningful interactions with facilitators, healthcare staff, and one another. In turn there are intuitive grounds for inferring that provision of enjoyable, stimulating activities will promote wellbeing, and qualitative analysis by CRILS (2014) has demonstrated that SR is associated with substantial feelings of engagement and pleasure. Part of this enjoyment may be linked with the way that the intervention provides a context for discussing personal experience, for it is established that individuals with semantic dementia retain a sense of self-image that is not purely characterised by contemporary experience (Larner, 2012). While failures in conventional memory were apparent (e.g., working, procedural, and semantic memory) residents would repeatedly link the poems with core parts of their personal selves that appeared to be retrieved via close attention and scrutiny to the poems' language. As one staff 
member remarked after attending an SR session "It is amazing - there is definitely something still there, and the poems really seem to bring it out" (CRILS, 2014, p.27).

In turn, this sense of spontaneous, episodic awareness could often be triggered through emotion than rather than memory. Unlike reminiscence therapy, SR creates a sense of 'presentness' (memory brought forward to the poem rather than back to an artefact, like a ration book), as well as harnessing the power of literature to evoke memories that may be a rich awakening force for ageing adults who have experienced much in life. This contrast was evident when a project worker brought in the same poem ('Daffodils') for discussion on different weeks. Some participants recalled it from previous sessions, whereas others remembered reading it at school; but whether participants remembered the poem from previous occasions or not, what was striking was that people frequently said different things in different ways about the same lines they selected, or picked out different parts of the poem. This indicated evidence of spontaneous newness in areas of emotional importance (rather than a set of routine responses as might have seemed characteristic of their condition), and to an extent is consistent with findings that older adults employ more elaborative processing for retrieving emotional material (Mather \& Knight, 2005), as well as indications that the affective context of a piece of text can beneficially effect memory performance in patients affected by Alzheimer's Disease (Boller et al., 2002; Moayeri et al., 2000). In turn, Moore and Davis's (2002) concept of 'quilting narrative' to assist communication amongst dementia patients includes the benefits of recognising and elaborating on information rather than recalling it, and it may be that the use of the same poems can serve this purpose. Effectively, the nature of memory investigated in this report is not short-term working memory or autobiographical stories (as recalled through reminiscence therapy). Rather, the use of memory in SR is to do with personal reawakening triggered by literature in significant emotional areas. Project workers believe that they can identify definable 'breakthrough' 
moments such as that described above during sessions. These qualitative findings may be quantified in future reports. It is currently estimated that in the first 20 sessions one such moment may be identified per session, rising to three after 25 sessions, and five after 45 . This requires further research. Nevertheless, the findings underline the importance of creative, interactive communication with older adults affected by dementia, as well as the ways in which such endeavours can be used to support self-expression and narrative agency (Fritsch et al., 2009; Hydén \& Örulv, 2009; Moore \& Davis, 2002). As Baldwin (2013) notes, "while the narratives of people living with dementia may at times appear...fragmented, inconsistent and incoherent, this may be seen as a function of an insistence of linear consistency and coherency rather than as inherently associated with dementia. We might, for instance, choose to reconfigure consistency and coherency to accommodate a sort of patchwork of fragments, individually uncertain in meaning or narrative value, into a meaningful whole" (pp.40-41).

Although financial value cannot be readily assigned to all health outcomes (Coast, 2004), interventions like SR offer value for money in terms of limited cost consequences and a corresponding positive impact (albeit in largely intangible terms) on residents, relatives, and staff (see CRILS, 2014). Whilst the conclusions that can be drawn from the current data are limited, our findings are consistent with an existing evidence base that demonstrates the valuable contribution of nonpharmacological interventions, specifically participatory artsbased strategies, for the care of dementia patients (Gerdner, 2000). Although further research is needed, existing work demonstrates that SR is associated with a reduction in neuropsychiatric symptoms (Billington et al., 2013); is able to utilise emotionally powerful literature to trigger 'awakenings' in people living with dementia; offer a richly attentive presentness in the activity and a sense of activated newness or renewal in the experience (which is different from mindfulness or reminiscence therapy); offer supportive social contact; and provide enjoyment, meaningfulness, and an enhancement of listening, memory 
and attention (Billington et al., 2013; CRILS, 2014). Correspondingly, the current results indicate that three months of reading group participation has a quantifiable impact on quality of life.

\section{Limitations}

The primary limitations of the project were the small sample, consequent lack of statistical power and generalizability, and the potential for clustering effects due to recruiting from four separate care homes. As such the results can only be interpreted cautiously until replicated with a larger sample and more rigorous design (e.g., a cluster-randomised controlled trial). In this respect a controlled trial, particularly one with participants matched for factors like medical history and social/cognitive functioning, would be better placed to estimate the extent to which positive effects are attributable to SR as opposed to other confounding variables. Finally, homogeneity within the groups resulted in low levels of psychopathological dementia symptoms and such ceiling effects limited the opportunity for positive change as a result of SR involvement. In this respect, it is also unclear whether the intervention has utility amongst individuals with more severe dementia than those in the current project with mild/moderate presentations.

\section{Acknowledgements}

This evaluation was commissioned by NHS North West to assess the effects of SR in care homes, developing out of the earlier report 'A Literature-Based Intervention for Older People Living with Dementia' commissioned by the Headley Trust.

\section{References}


Alzheimer's Disease International. (2011). Read with people with moderate to advanced dementia. Retrieved from: http://www.alz.co.uk/icaniwill/library/professionalcarers/activities/reading

Alzheimer's Disease International (ADI). (2012). Policy Brieffor Heads of Government: The Global Impact of Dementia 2013-2050. ADI: London.

Baldwin, C. (2013). Narrative Social Work: Theory and Application. Policy Press: Bristol. Billington, J. (2011). “'Reading for life': Prison reading groups in practice and theory." Critical Survey, Vol.23 No.3, pp. 67-85.

Billington, J., Carroll, J., Davis, P., Healey, C., \& Kinderman, P. (2013). “A literature-based intervention for older people living with dementia." Perspectives in Public Health, Vol. 133 No.3, pp.165-173.

Billington, J., Humphreys, A.L, Jones, A., \& McDonnell, K. (2014). “A literature-based intervention for people with chronic pain." Arts \& Health. Advanced online publication. Doi: 10.1080/17533015.2014.957330

Boller, F., Dequeker, J., Degreef, H., El Massioui, F., Devouche, E., Busschots, A. M., ... \& Starkstein, S. E. (2002). "Processing emotional information in Alzheimer's disease: Effects on memory performance and neurophysiological correlates." Dementia and Geriatric Cognitive Disorders, Vol. 14 No.2, pp. 104-112.

Centre for Research into Reading, Literature and Society (CRILS). (2014). Read to Care: An Investigation into Quality of Life Benefits of Shared Reading Groups for People Living with Dementia, University of Liverpool, Liverpool.

Coast, J. (2004). "Is economic evaluation in touch with society’s health values?” British Journal of Medicine, Vol.329 No.7476, pp.1233-1236.

Davis, P. (2008). “Syntax and pathways.” Interdisciplinary Science Reviews, Vol. 33 No.4, pp.265-277. 
Davis, P., Keidel, J.L., Gonzalez-Diaz, V., Martin, C.D., \& Thierry, G. (2013). “How

Shakespeare tempests the brain: Neuroimaging insights." Cortex, Vol.49 No.4, pp.2164.

Department of Health (DoH). (2012a). Living Well with Dementia: A National Dementia Strategy. London: DoH.

Department of Health (DoH). (2012b). Prime Minister's Challenge on Dementia: Delivering Major Improvements in Dementia Care and Research by 2015. London: DoH.

Dowrick, C., Billington, J., Robinson, J., Hamer, A., \& Williams, C. (2012). “Get into Reading as an intervention for common mental health problems: exploring catalysts for change." Medical Humanities, Vol.38 No.1, pp. 15-20.

Fritsch, T., Kwak, J., Grant, S., Lang, J., Montgomery, R.R., \& Basting, A.D. (2009). "Impact of TimeSlips, a creative expression intervention program, on nursing home residents with dementia and their caregivers." The Gerontologist, Vol. 49 No. 1, pp.117-127.

George, D.R. \& Houser, W.S. (2014). "I'm a storyteller!”: Exploring the benefits of TimeSlips creative expression program at a nursing home. American Journal of Alzheimer's Disease and Other Dementias, Vol.29 No.8, pp.678-84.

Gerdner, L.A. (2000). "Music, art, and recreational therapies in the treatment of behavioral and psychological symptoms of dementia.” International Psychogeriatrics, Vol. 12 Sup. S1, pp.359-366.

Hodge, S., Robinson, J., \& Davis, P. (2007). "Reading between the lines: The experiences of taking part in a community reading project.” Medical Humanities, Vol.33 No.2, pp. $100-104$.

Hydén, L. C., \& Örulv, L. (2009). "Narrative and identity in Alzheimer's disease: A case study." Journal of Aging Studies, Vol. 23 No.4, pp. 205-214. 
Juncos-Rabadan, O., Pereiro, A.X., Facal, D., Rodriguez, N., Lojo, C., Caamaño, J.A ... Eiro, P. (2012). "Prevalence and correlates of cognitive impairment in adults with subjective memory complaints in primary care centres." Dementia and Geriatric Cognitive Disorders, Vol.33 No.4, pp.226-232.

Kaufer, D.I., Cummings, J.L., Ketchel, P., Smith, V., MacMillan, A., Shelley, T., ... \& DeKosky, S.T. (2000). "Validation of the NPI-Q, a brief clinical form of the Neuropsychiatric Inventory." The Journal of Neuropsychiatry and Clinical Neurosciences, Vol.12 No.2, pp. 233-239.

Kinney, J.M., \& Rentz, C.A. (2005). “Observed well-being among individuals with dementia: Memories in the Making, an art program, versus other structured activity. American Journal of Alzheimer's Disease and Other Dementias, Vol. 20 No. 4, pp.220-227. Larner, A.J. (2012). Dementia in Clinical Practice: A Neurological Perspective: Studies in the Dementia Clinic. London: Springer Publishing.

Longden, E., Davis, P., Billington, J., Lampropoulou, S., Farrington, G., Magee, F., Walsh, E., \& Corcoran, R. (2015). "Shared Reading: Assessing the intrinsic value of a literaturebased health intervention." Medical Humanities. In press.

Low, L.F., Brodaty, H., Goodenough, B., Spitzer, P., Bell, J.P., Fleming, R., ... \& Chenoweth, L. (2013). “The Sydney Multisite Intervention of LaughterBosses and ElderClowns (SMILE) study: Cluster randomised trial of humour therapy in nursing homes." British Medical Journal Open, Vol. 3 No. 1, e002072.

Mather, M., \& Knight, M. (2005). "Goal-directed memory: The role of cognitive control in older adults' emotional memory." Psychology and Aging, Vol.20 No.4, pp. 554-570.

Moore, L. A., \& Davis, B. (2002). “Quilting narrative: Using repetition techniques to help elderly communicators." Geriatric Nursing, Vol.23 No.5, pp.262-266. 
Moayeri, S. E., Cahill, L., Jin, Y., \& Potkin, S. G. (2000). "Relative sparing of emotionally influenced memory in Alzheimer's disease.” Neuroreport, Vol.11 No.4, pp.653-655.

Philips, L.J., Reid-Arndt, S.A., \& Pak, Y. (2010). "Effects of a creative expression intervention on emotions, communication, and quality of life in persons with dementia." Nursing Research, Vol.59 No.6, pp.417-425.

Robinson J. (2008). Reading and Talking: Exploring the Experience of Taking Part in Reading Groups in Walton Neuro-Rehabilitation Unit. Liverpool: HaCCRU Research Report 114/08 .

Rowen, D., Mulhern, B., Banerjee, S., van Hout, B., Young, T. A., Knapp, M., ... \& Brazier, J.E. (2012). "Estimating preference-based single index measures for dementia using DEMQOL and DEMQOL-Proxy." Value in Health, Vol. 15 No. 2, pp. 346-356.

Smith, S.C., Lamping, D.L., Banerjee, S., Harwood, R., Foley, B., Smith, P., ... \& Knapp, M. (2005). "Measurement of health-related quality of life for people with dementia: development of a new instrument (DEMQOL) and an evaluation of current methodology." Health Technology Assessment (Winchester, England), Vol. 22 No. 10, pp. 1-93.

Svansdottir, H.B., \& Snaedal, J. (2006). "Music therapy in moderate and severe dementia of Alzheimer's type: A case-control study.” International Psychogeriatrics, Vol. 18 No. 04, pp.613-621.

Staricoff, R.L. (2004). Arts in Health: A Review of the Medical Literature. London: Arts Council England.

Thierry, G., Martin, C.D., Gonzalez-Diaz, V., Rezaie, R., Roberts, N., \& Davis P. (2008). "Event-related potential characterisation of the Shakespearean functional shift in narrative sentence structure." NeuroImage, Vol. 40 No. 2, pp.923-931. 
Van der Roest, H.G., Meiland, F.J.M., Maroccini, R., Comijs, H.C., Jonker, C., \& Droumles R.M. (2007). "Subjective needs of people with dementia: A review of the literature." International Psychogeriatrics, Vol.19 No.3, pp.559-592.

Wilson, R.S., Boyle, P.A., Yu, L., Barnes, L.L., Schneider, J.A., \& Bennett, D.A. (2013). "Life-span cognitive activity, neuropathologic burden, and cognitive aging." Neurology, Vol.81 No.4, pp.314-321. 
Table 1. Mean (standard deviation) DEMQOL-Proxy scores for residents according to reading and waiting conditions.

\begin{tabular}{|c|c|c|c|c|c|c|c|c|}
\hline Group frequency & $\mathrm{N}$ & $\begin{array}{l}\mathrm{B} \\
\mathrm{a} \\
\mathrm{s} \\
\mathrm{el} \\
\mathrm{i} \\
\mathrm{n} \\
\mathrm{e}\end{array}$ & Month 1 & $\begin{array}{l}\text { Month } \\
2\end{array}$ & Month 3 & Month 4 & $\begin{array}{l}\text { Mont } \\
\text { h } 5\end{array}$ & Month 6 \\
\hline
\end{tabular}

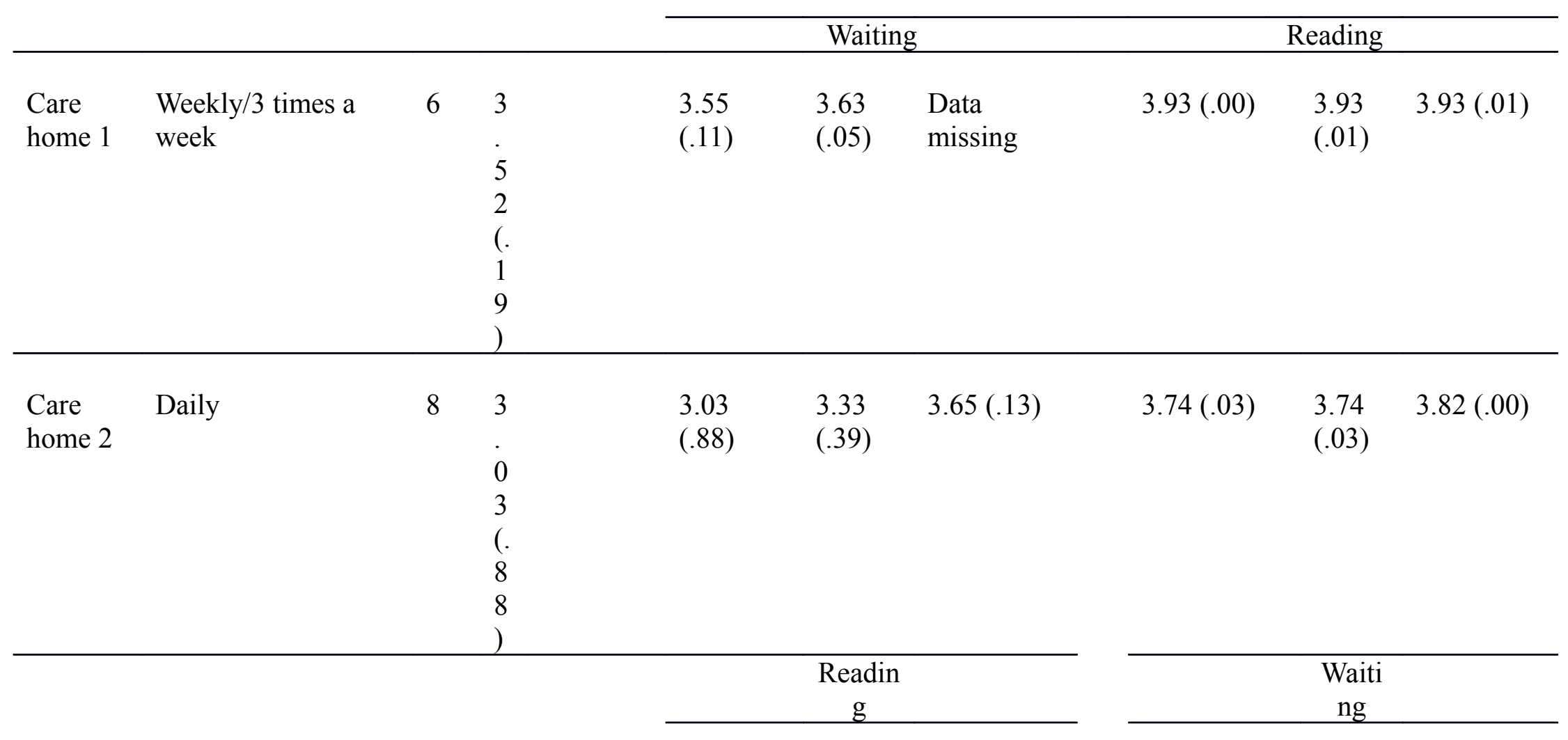




\begin{tabular}{|c|c|c|c|c|c|c|c|c|c|}
\hline $\begin{array}{l}\text { Care } \\
\text { home } 3\end{array}$ & Weekly & 10 & $\begin{array}{l}3 \\
. \\
7 \\
3 \\
(. \\
0 \\
0 \\
0\end{array}$ & $\begin{array}{l}3.73 \\
(.01)\end{array}$ & $\begin{array}{l}3.75 \\
(.01)\end{array}$ & $3.76(.00)$ & $3.82(.01)$ & $\begin{array}{l}3.82 \\
(.00)\end{array}$ & $3.82(.01)$ \\
\hline $\begin{array}{l}\text { Care } \\
\text { home } 4\end{array}$ & Daily & 7 & $\begin{array}{l}3 \\
. \\
3 \\
0 \\
(. \\
1 \\
6 \\
)\end{array}$ & $\begin{array}{l}3.90 \\
(.09)\end{array}$ & $\begin{array}{l}3.90 \\
(.09)\end{array}$ & $3.98(.03)$ & $3.99(.02)$ & $\begin{array}{l}3.95 \\
(.07)\end{array}$ & $3.90(.23)$ \\
\hline
\end{tabular}

Note. Due to unforeseen circumstances, care home 1 was recruited a month later then the remaining homes. Hence their baseline scores are equivalent to month 1 for the other care homes. However, as participants were in the waiting condition, this meant baseline data could still be collected at month 1 and every month thereafter. 